\title{
Revisão de literatura sobre o atendimento ao paciente surdo pelos profissionais da saúde
}

\author{
LITERATURE REVISION ABOUT THE ATTENDANCE OF DEAF PATIENT BY HEALTH \\ PROFESSIONALS
}

REVISIÓN DE LA BIBLIOGRAFÍA SOBRE LA ATENCIÓN DE LOS PROFESIONALES DE SALUD AL PACIENTE SORDO

Neuma Chaveiro ${ }^{1}$, Maria Alves Barbosa ${ }^{2}$, Celmo Celeno Porto ${ }^{3}$

\section{RESUMO}

O objetivo deste estudo de revisão sistemática da literatura é analisar e identificar a comunicação entre o paciente surdo e o profissional da saúde, bem como investigar a assistência oferecida. Foram utilizadas as bases de dados informatizadas para a coleta de dados, tendo como palavraschave os termos paciente, surdo e comunicação. Os resultados foram agrupados em três categorias: comunicação, formação dos profissionais da saúde e aspectos legais. A categoria comunicação aborda barreiras comunicativas, linguagem escrita e a presença do intérprete. Na segunda categoria, o foco está na formação dos profissionais referentes à comunidade surda. A terceira categoria relata os aspectos legais no atendimento ao paciente surdo. A revisão mostrou que há barreiras de comunicação entre paciente surdo e profissional da saúde, e que o atendimento é um desafio para ambos. Ressalta também a necessidade de formação sobre o paciente surdo.

\section{DESCRITORES}

Comunicação.

Surdez.

Linguagem de sinais.

Relações profissional-paciente.

\begin{abstract}
The purpose of this systematic literature review is to analyze and identify the communication between deaf patients and health professionals, as well as to investigate the care offered. The computerized databases were used for data collection, using the keywords paciente (patient), surdo (deaf person), and comunicação (communication). The results were grouped in three categories: communication, health professional education, and legal aspects. The communication category deals with communicative barriers, written language and the presence of the interpreter. In the second category, the focus is on the education of the professionals in relation to the deaf community. The third category reports the legal aspects involved in caring for deaf patients. The review showed that there are communication barriers between deaf patients and health professionals, and that health care is a challenge for both. It also emphasized that there is a need for education regarding the deaf patients.
\end{abstract}

\section{KEYWORDS}

Communication.

Deafness.

Sign language.

Professional-patient relations.

\section{RESUMEN}

El objetivo de este estudio de revisión sistémica es analizar e identificar la comunicación entre el paciente sordo y el profesional de salud, así como investigar la atención brindada. Fueron utilizadas las bases de datos informatizadas para la recolección de datos, siendo las palabras claves paciente, surdo y comunicação. Los resultados fueron agrupados en tres categorías: comunicación, formación de los profesionales de salud y aspectos legales. La categoría comunicación trata sobre las barreras comunicativas, el lenguaje escrito y la presencia del intérprete. En la segunda categoría el enfoque fue dado a la formación de los profesionales con respecto a la población sorda. Finalmente, la tercera categoría referida a los aspectos legales en la atención al paciente sordo. Esta revisión demostró que existen barreras comunicativas entre el paciente sordo y el profesional de salud. Asimismo, la atención es un reto para ambos, destacando la necesidad de invertir en la formación de profesionales para prestar cuidados al paciente sordo.

\section{DESCRIPTORES}

Comunicación.

Sordera.

Lenguaje de signos.

Relaciones profesional-paciente.

${ }^{1}$ Mestranda do Programa de Pós-Graduação em Ciências da Saúde da Universidade Federal de Goiás (UFG). Fonoaudióloga do Centro Estadual de Apoio ao Deficiente do Estado de Goiás (CEAD). Intérprete da Língua Brasileira de Sinais - LIBRAS. Goiânia, GO, Brasil. neumachaveiro@ig.com.br 2 Professora Doutora Adjunta III da Faculdade de Enfermagem, Universidade Federal de Goiás (UFG). Goiânia, GO, Brasil. malves@ih.com.br ${ }^{3}$ Professor Emérito da Faculdade de Medicina, Universidade Federal de Goiás (UFG). Coordenador do Programa de Pós-Graduação em Ciências da Saúde da Universidade Federal do Goiás (UFG). Goiânia, GO, Brasil. celeno@cardiol.br

$\begin{aligned} & \begin{array}{r}\text { Rev Esc Enferm USP } \\ 2008 ; 42(3): 578-83 .\end{array} \begin{array}{l}\text { Recebido: 19/09/2006 } \\ \text { www.ee.usp.br/reeusp/ }\end{array} \\ & \text { Aprovado: 27/04/2007 }\end{aligned}$




\section{INTRODUÇÃO}

O censo do IBGE (Instituto Brasileiro de Geografia e Estatística/2000) revela que há no Brasil 24,5 milhões de pessoas com deficiência, o que corresponde a $14,5 \%$ da população. Destes, 16,7\% apresentam deficiência auditiva, ou seja, existem no Brasil 5.735.099 (cinco milhões setecentos e trinta e cinco mil e noventa e nove) surdos. Levando-se em conta o crescimento anual da população, teríamos, a cada ano, no Brasil, aproximadamente 93.295 (noventa e três mil duzentas e noventa e cinco) crianças com surdez $z^{(1)}$.

A surdez era, antigamente, algo discriminador, os indivíduos surdos considerados dignos de pena e vítimas da incompreensão de toda a sociedade e até mesmo da própria família ${ }^{(2)}$. Contudo essa visão vem se modificando e, atualmente, é discutida por profissionais de diversas áreas do conhecimento.

Helen Keller que ficou surda-cega desde os 19 meses de idade, considerava que ser surdo é pior do que ser cego, porque ser cego isola a pessoa das coisas, mas ser surdo isola a pessoa das outras pessoas. Ela nasceu em 1880, escreveu vários livros e ficou famosa em virtude do livro The World I Live In, publicado em $1914^{(3)}$.

Vale ressaltar o fato da surdez causar tantas conseqüências, embora a pessoa surda tenha um meio de comunicação espaço-visual tão competente quanto o oral-auditivo, utilizado pelas pessoas ouvintes. O problema de comunicação do indivíduo surdo não é orgânico, e sim, social e cultural.

A relação dos profissionais de saúde com pacientes que têm audição em níveis normais é estabelecida pelo código verbal, mecanismo normalmente não utilizado pelos pacientes surdos, que recorrem à linguagem de sinais para se comunicar ${ }^{(4)}$. Elas são de modalidade espaço-visual, pois os signos compartilhados são recebidos pelos olhos e sua produção realizada pelas mãos no espaço. São reconhecidas como línguas pela lingüística, que lhes atribui o conceito de língua natural e não as considera como problema do surdo ou como patologia da linguagem ${ }^{(2)}$.

As línguas de sinais estão presentes nos cinco continentes, mas não são universais, cada uma tem sua própria estrutura gramatical, sendo que com as línguas de sinais é possível expressar qualquer conceito complexo, sutil ou abstrato. As línguas de sinais são um sistema lingüístico altamente estruturado e tão complexo como as línguas faladas, estruturando-se neurologicamente nas mesmas áreas cerebrais das línguas orais ${ }^{(5-6)}$.

Para a pessoa surda, o meio de comunicação utilizado pelo meio que a cerca, não se apresenta como um recurso que vem facilitar seu intercâmbio com o mundo, mas um obstáculo que precisa transpor com dificuldades para chegar ao mundo social de forma efetiva ${ }^{(7)}$.
Diante de todo o complexo que envolve a relação entre paciente surdo e profissional da saúde, surgem duas questões: sendo a comunicação uma ferramenta essencial nos mais variados procedimentos na área da saúde, como os profissionais interagem com os pacientes surdos? As barreiras de comunicação que surgem entre duas línguas diferentes, a língua de sinais e a língua oral, comprometem a qualidade da assistência oferecida à população surda? A proposta deste estudo é responder a essas perguntas investigando a produção científica, com o objetivo de identificar e analisar estudos sobre a comunicação entre paciente surdo e profissional da saúde e o atendimento prestado à população surda.

\section{MÉTODO}

Artigo de revisão sistemática da literatura cuja busca foi realizada em janeiro de 2007 na Biblioteca Virtual em Saúde - BVS, utilizando-se as bases de dados da Literatura da América Latina e do Caribe em Ciências da Saúde (LILACS), da Literatura Internacional (MEDLINE), Saúde na Adolescência (ADOLEC) e Base de dados de Enfermagem (BDENF).

Na base de dados MEDLINE, optou-se pela revisão no período de 1996 a 2006, foi utilizada a pesquisa estruturada por meio do formulário básico com os termos paciente, surdo e comunicação como palavras-chave. Foram encontrados um total de 65 artigos.

Nas bases LILACS, ADOLEC e BDENF, optou-se pela revisão de literatura sem restrição de período e procedeu-se a uma pesquisa estruturada utilizando o formulário básico com os mesmos termos usados anteriormente. Foram encontradas na LILACS um total de 5 artigos, sendo que 1 se repete na MEDLINE. Na ADOLEC e BDENF foram identificados 2 artigos em cada, sendo que eles se repetiam na LILACS e MEDLINE.

Todos os 70 artigos foram lidos para identificação dos objetivos propostos e excluídos 16 artigos da MEDLINE, 2 da LILACS pois abordavam aspectos terapêuticos e cirúrgicos. Consoante com os objetivos do estudo, portanto, encontramos 51 artigos.

Realizou-se também busca em sítios na Internet de organizações que trabalham com a comunidade surda. Os dados encontrados deram origem a três categorias temáticas: comunicação, formação dos profissionais da saúde e aspectos legais.

\section{RESULTADOS E DISCUSSÃO}

Os trabalhos foram agrupados em três categorias temáticas: comunicação, formação dos profissionais da saúde e aspectos legais. Na primeira categoria (comunicação) foram selecionados trabalhos sobre barreiras comunica- 
tivas, linguagem escrita, comunicação não-verbal e a presença do intérprete da língua de sinais como mediador. $\mathrm{Na}$ segunda categoria (formação dos profissionais) foram selecionados trabalhos que discutiam a importância de programas e formação dos profissionais e estudantes a cerca da identidade e aspectos socioculturais da comunidade surda. Na terceira categoria (aspectos legais) foram selecionados trabalhos que relatavam os aspectos legais no atendimento ao paciente surdo.

Os 51 artigos selecionados de acordo com objetivos propostos deste estudo, foram agrupados nas categorias a seguir, podendo um artigo estar incluso em mais de uma categoria.

Quadro 1 - Distribuição dos artigos publicados na MEDLINE e LILACS, segundo as categorias estabelecidas - São Paulo - 2007

\begin{tabular}{|l|c|c|c|}
\hline Fonte de indexação / Categorias* & Comunicação & $\begin{array}{c}\text { Formação dos } \\
\text { Profissionais }\end{array}$ & Aspectos legais \\
\hline MEDLINE & 35 & 20 & 12 \\
\hline LILACS & 2 & 1 & - \\
\hline Total de artigos* & 37 & 21 & 12 \\
\hline
\end{tabular}

Apesar do significativo número (51) de trabalhos publicados no período estudado, sobre a comunicação entre paciente surdo e profissional da saúde e a assistên-

cia prestada a essa população, a produção científica brasileira é ainda muito incipiente constando apenas 2 trabalhos.

Quadro 2 - Distribuição dos artigos publicados sobre o atendimento do paciente surdo pelos profissionais da saúde, de acordo com ano, país e idioma publicado - São Paulo - 2007

\begin{tabular}{|l|c|c|c|c|c|c|c|c|c|c|c|c|c|}
\hline $\begin{array}{c}\text { País - } \\
\text { Idioma / Ano }\end{array}$ & 1992 & 1996 & 1997 & 1998 & 1999 & 2000 & 2001 & 2002 & 2003 & 2004 & 2005 & 2006 & Total \\
\hline E.U.A. & - & 4 & 2 & - & 6 & 5 & - & 5 & 1 & 3 & 2 & 2 & 30 \\
\hline Inglaterra & - & 1 & 1 & 1 & 2 & 1 & - & 1 & - & 1 & 3 & 1 & 12 \\
\hline Alemanha & - & - & - & - & - & - & - & - & 1 & - & 1 & - & 2 \\
\hline Escócia & - & - & - & - & - & 1 & - & - & - & - & - & - & 1 \\
\hline Suíça & - & - & - & - & - & - & - & - & - & - & - & - & 1 \\
\hline Arábia Saudita & - & - & - & - & - & 1 & - & - & - & - & - & - & 1 \\
\hline França & - & - & - & - & - & - & 1 & - & - & - & - & - & 1 \\
\hline Brasil & - & - & - & - & - & - & - & - & - & - & - & 1 & 1 \\
\hline \hline Português & - & - & - & - & - & - & - & - & 1 & - & - & - & 1 \\
\hline Espanhol & 1 & - & - & - & - & - & - & - & - & - & - & - & 1 \\
\hline \hline Total & 1 & 5 & 3 & 1 & 8 & 9 & 1 & 6 & 3 & 4 & 6 & 4 & 51 \\
\hline
\end{tabular}

Nota: MEDLINE - De acordo com o ano (1996-2006) e o país de publicação.

No resultado apresentado a seguir é importante ressaltar que a revisão da literatura serviu como um balizador para a construção do texto, dentro das categorias temáticas já mencionadas.

\section{Comunicação}

A comunicação é uma importante ferramenta para os profissionais da saúde no diagnóstico e no tratamento, pois faz parte deles instruções verbais de variados proce-
LILACS - De acordo com o idioma publicado, não informa país.

dimentos, cujo resultado pode ser comprometido pela não compreensão das partes envolvidas ${ }^{(8-10)}$.

As pesquisas mostram a necessidade de uma melhor comunicação dos profissionais com os pacientes surdos, no entanto a comunicação com as pessoas surdas continua negligenciada nos sistemas de saúde ${ }^{(8,10-11)}$. Por isso, a linguagem não-verbal é um recurso de comunicação que precisa ser conhecido e valorizado na prática das ações em saúde ${ }^{(12)}$. Mesmo que não se conheça a língua de sinais, 
é fundamental interpretar seus aspectos suprassegmentais que incluem gestos, expressões faciais e corporais $^{(13)}$.

A comunicação não-verbal é de extrema importância no atendimento aos pacientes e permite a excelência do cuidar em saúde, o profissional que a reconhece adequadamente remete significados aos sinais não-verbais dos pacientes favorecendo a interação ${ }^{(12,14)}$.

Comparando-se as pessoas surdas e as que ouvem, verificam-se claramente suas particularidades, que são fundamentais na intervenção com pacientes surdos. Não é possível generalizar os surdos, como se todos fossem iguais, pois há diferenças em termos sensoriais e comunicativos ${ }^{(15)}$.

Fatores que devem ser considerados nessa população incluem o grau da perda auditiva, a época em que ocorreu o déficit auditivo, as preferências lingüísticas, assim como questões psicológicas e sócio-culturais ${ }^{(7)}$.

Alguns estudos têm como suporte equivocado considerar que as pessoas são semelhantes, não observando na prática a sua diversidade, pois cada paciente é um caso, portanto generalizar os estudos e canalizar como se todos fossem um, é, no mínimo, uma ingenuidade. Para evitar esta postura é necessário que o profissional da saúde deixe de considerar apenas a doença e se aplique em cuidar de uma pessoa que, circunstancialmente, está doente e que é única ${ }^{(16)}$.

Imagina-se que a presença do intérprete nos serviços de saúde solucionaria todos os problemas de comunicação entre o paciente e o profissional de saúde, entretanto, verifica-se que nem sempre é assim que ocorre. A atuação do intérprete pode melhorar, mas não é decisiva para um atendimento de qualidade ${ }^{(17)}$. Os surdos valorizam a presença do intérprete, mas com algumas ressalvas: desconfiança, constrangimento de se expor frente ao intérprete, sentimento de piedade e dificuldade de encontrar intérpretes disponíveis $^{(14,18)}$.

Como os surdos com freqüência não apresentam problema visual, a escrita poderia ser uma alternativa, mas na realidade não é assim; isto porquê, para eles, a língua oral-auditiva do seu país é uma segunda língua, e como qualquer língua estrangeira, seu aprendizado é difícil. Desde modo, a escrita não é o caminho para melhorar o atendimento $^{(14)}$.

O sucesso na comunicação com os pacientes surdos depende das características individuais de cada pessoa surda e, em relação às necessidades comunicativas, eles podem ser divididos em três categorias:

1. Pessoas com dificuldades de audição e que lingüisticamente ainda se beneficiam do código verbal.

2. Pessoas surdas que se comunicam oralmente, que por sua vez, podem ser divididas em dois grupos: a) adultos que perderam a audição; b) crianças surdas que foram educadas numa linha oralista.
3. Pessoas surdas que se comunicam pela língua de sinais que perderam a audição antes dos três anos de idade, ou seja, no período de aquisição da linguagem verbal, e tiveram como meio de comunicação a língua de $\operatorname{sinais}^{(9)}$.

A população de surdos é um grupo heterogêneo e inclui pessoas que têm vários graus de perda auditiva, utilizamse de diversos meios para se comunicar e pertencem a diferentes culturas ${ }^{(8)}$. No atendimento ao paciente surdo tornase importante conhecer essas diferenças que influenciarão no vínculo estabelecido.

A barreira de comunicação que dificulta ou até impede a interação entre surdos e profissionais de saúde só pode ser superada quando se encontram formas de interação, única maneira de se garantir uma assistência de boa qualidade ${ }^{(5-6)}$.

\section{Formação dos profissionais da saúde}

Há muitas barreiras no atendimento ao paciente surdo, destacando-se as dificuldades lingüísticas, falta de confiança no mundo dos que ouvem, atribuir aos surdos baixa inteligência comparando-os com deficientes mentais e falta de acesso dos surdos às informações preventivas $^{(19)}$. As pessoas surdas têm também pouco conhecimento da assistência em saúde, incluindo menor compreensão dos programas preventivos como HIV/AIDS e visitam com menor freqüência os médicos, comparados com as pessoas que ouvem ${ }^{(8,20-21)}$.

O encontro com o paciente surdo pode ser esporádico, mas o desafio para os profissionais da saúde está além dos serviços especializados. Habilidades no trabalho com pessoas que não partilham a língua oral e apresentam cultura própria não são rotineiramente ensinadas, por isso os profissionais podem não estar preparados para o encontro com o paciente surdo ${ }^{(22-24)}$.

Para o profissional de saúde uma efetiva comunicação com seus clientes propicia um atendimento de melhor qualidade ${ }^{(5,25-26)}$, portanto a capacitação dos profissionais da saúde, para atender esses pacientes é uma necessidade urgente, uma formação que contemple os métodos de comunicação, cultura surda, noções básicas de língua de sinais e leitura-labial e como se posicionar frente ao atendimento do surdo, assegura o acesso aos cuidados de saúde ${ }^{(19,21,23,27-28)}$.

Estudos mostram que para trabalhar com grupos minoritários é essencial compreender sua cultura, a população surda que usa a língua de sinais, é lingüística e culturalmente um grupo minoritário ${ }^{(24,29)}$, mas a maioria dos cursos na área de saúde caracterizam a surdez apenas como condição patológica não compreendendo a população surda como um grupo minoritário ${ }^{(23)}$.

A comunidade surda é composta por pessoas que usam a língua de sinais como primeiro meio de comunicação, possuem sentimento de pertencimento à cultura surda, com características únicas, linguagem e normas sociais. 
Diferem-se de outras deficiências em virtude dos processos comunicativos e não pela deficiência física(6).

Estudos culturalistas sobre surdez elegeram a língua de sinais como fator primordial da cultura surda, sendo respaldado pela lingüística que a considera língua materna da comunidade surda, apresentando todas as características que lhe confere o valor de língua. Estabelece assim as fronteiras políticas do movimento cultural dos surdos ${ }^{(29)}$.

\section{Aspectos legais}

A partir de uma visão inclusiva a Organização Mundial de Saúde apresentou em 2003 um novo modelo de avaliação das deficiências, Classificação Internacional de Funcionalidade - CIF. Até então as pessoas com deficiências eram avaliadas apenas pelos parâmetros do Código Internacional de Doenças - CID. O CID faz aferição sob o ângulo da enfermidade e o CIF realiza sua avaliação a partir da funcionalidade. A adoção do CIF não elimina o uso do CID. Cumpre salientar que o Brasil assinou acordo, juntamente com outros países, comprometendo-se a adotar oficialmente esta nova referência a partir do ano de $2004^{(30)}$.

Admitindo-se a complexidade da relação do médico com pacientes surdos, torna-se necessário conhecer os aparatos legais que a regem, bem como compreender a identidade da pessoa surda e os fatores culturais que caracterizam a comunidade surda, sendo estes os diferenciais essenciais na qualidade dos serviços prestados a essa população(31).

A partir do momento em que os países têm reconhecido oficialmente a língua de sinais como língua natural da comunidade surda, o profissional da saúde está sendo requisitado pela lei para assegurar efetivo atendimento a população surda ${ }^{(10-11,32)}$. De acordo com o Decreto $\mathrm{n}$ - 5.626, de 22 de dezembro de 2005, que regulamenta a lei no 10.436/ 02 - Lei da Língua Brasileira de Sinais - LIBRAS, destaca para a área da saúde o Capítulo VII da garantia do direito à saúde das pessoas surdas ou com deficiência auditiva; neste Decreto o artigo 25 enuncia:

A partir de um ano da publicação deste Decreto, o Sistema Único de Saúde - SUS - e as empresas que detêm concessão ou permissão de serviços públicos de assistência à saúde, na perspectiva da inclusão plena das pessoas surdas ou com deficiência auditiva em todas as esferas da vida social, devem garantir a atenção integral à saúde, nos diversos níveis de complexidade e especialidades médicas, efetivando:

IX - atendimento às pessoas surdas ou com deficiência auditiva na rede de serviços do SUS e das empresas que detêm concessão ou permissão de serviços públicos de assistência à saúde, por profissionais capacitados para o uso de LIBRAS ou para sua tradução e interpretação; e

$X$ - apoio à capacitação e formação de profissionais da rede de serviços do SUS para o uso de LIBRAS e sua tradução e interpretação ${ }^{(33)}$.
Nesta linha de ação o Ministério da Saúde elaborou o manual A Pessoa com Deficiência e o Sistema Único de Saúde, destinado aos médicos, enfermeiros e outros profissionais das equipes de saúde, no qual propõe a inclusão social das pessoas com deficiência como meta mais abrangente. Entre as informações contidas neste manual destaca-se a seguinte:

A atenção integral à saúde, destinada à pessoa com deficiência, pressupõe uma assistência específica à sua condição, ou seja, serviços estritamente ligados à sua deficiência, além de assistência a doenças e agravos comuns a qualquer cidadão ${ }^{(34)}$.

A aprovação da Lei Federal no 10.436/02 resultou da luta da comunidade surda brasileira que utiliza a língua de sinais. Cabendo às instituições públicas oportunizar programas que visem à formação dos profissionais da área de saúde no atendimento e tratamento dos pacientes surdos ${ }^{(35)}$.

A Língua de Sinais não é uma escolha, é a língua da comunidade surda. $\mathrm{O}$ acesso à informação nesta língua não está bem definido, pois existem barreiras que dificultam a compreensão das doenças e as decisões referentes a saúde ${ }^{(31,36)}$. Estudo realizado nos Estados Unidos, verificou-se que os pacientes surdos preferem ser atendidos por médicos que sabem a Língua de Sinais ou médicos que são $\operatorname{surdos}^{(31,36)}$.

\section{CONCLUSÃO}

A revisão de literatura mostrou que as barreiras de comunicação entre paciente surdo e profissional da saúde, podem colocar em risco a assistência prestada, podendo prejudicar o diagnóstico e o tratamento.

Os estudos demonstraram que é fundamental conhecer as particularidades culturais e lingüísticas da comunidade surda de modo a favorecer a interação entre paciente e profissional, reduzindo significativamente o desconforto de ambos nos encontros clínicos.

Durante a leitura dos artigos científicos verificamos que o atendimento do paciente surdo é um desafio para os profissionais da saúde e para o próprio surdo, pois a comunicação verbal não é um recurso que facilita o intercâmbio da pessoa surda com o mundo, mas, pelo contrário, um obstáculo que ela precisa transpor para chegar ao mundo social de forma efetiva.

As pesquisas sugerem, principalmente as instituições acadêmicas, oportunizar estudos com questões referentes ao atendimento do paciente surdo, reconhecendo que estar diante de uma pessoa surda não implica apenas constatar um déficit auditivo, mas envolve também aspectos socioculturais e legais.

Sugere-se a realização de estudos referentes ao atendimento do paciente surdo na área da saúde, haja vista o número reduzido de publicações científicas no Brasil. 


\section{REFERÊNCIAS}

1. Instituto Brasileiro de Geografia e Estatística (IBGE). Censo Demográfico Brasileiro, 2000 [texto na Internet]. Rio de Janeiro; 2000. [citado 2006 jan. 10]. Disponível em: http://www.ibge.gov.br

2. Sacks O. Vendo vozes: uma viagem ao mundo dos surdos. Trad. de Laura Teixeira Motta. São Paulo: Companhia das Letras; 1998.

3. Reis VPF. A linguagem e seus efeitos no desenvolvimento cognitivo e emocional da criança surda. Rev Espaço Inf Técnico-Científico INES. 1997;6(1):23-39.

4. Lezzoni LI, O'Day BL, Killeen M, Harker H. Communicating about health care: observations from persons who are deaf or hard of hearing. Ann Intern Med. 2004;140(5):356-62.

5. Barbosa MA, Oliveira MA, Siqueira KM, Damas KCA, Prado MA. Linguagem Brasileira de Sinais: um desafio para a assistência de enfermagem. Rev Enferm UERJ. 2003;11(3):247-51.

6. Allen B, Meyers N, Sullivan J, Sullivan M. American Sign Language and end-of-life care: research in the deaf community. HEC Forum. 2002;14(3):197-208.

7. Cardoso AH, Rodrigues KG, Bachion MM. Perception of with severe or profound deafness about the communication process during health care. Rev Lat Am Enferm. 2006;14(4):553-60.

8. Meador HE, Zazove P. Health care interactions with deaf culture. J Am Board Fam Pract. 2005;18(3):218-22.

9. Barnett S. Communication with deaf and hard-of-hearing people: a guide for medical education. Acad Med. 2002; 77(7):694-700.

10. Ralston E, Zazove P, Gorenflo DW. Physicians' attitudes and beliefs about deaf patients. J Am Board Fam Pract. 1996;9(3):167-73.

11. Hudson R. Including deaf patients in the conversation. Fam Pract Manag. 2004;11(6):37-40.

12. Silva MF, Silva MJP. A auto-estima e o não-verbal dos pa-cientes com queimaduras. Rev Esc Enferm USP. 2004;38(2):206-16.

13. Chaveiro N, Barbosa MA. A surdez, o surdo e seu discurso. Rev Eletrônica Enferm [periódico na Internet]. 2004 [citado 2007 jan. 15];6(2):[cerca de 6 p.]. Disponível em: http://www.fen.ufg.br/revista/revista6_2/surdez.html

14. Chaveiro N, Barbosa MA. Assistência ao surdo na área de saúde como fator de inclusão social. Rev Esc Enferm USP. 2005;39(4):417-22.

15. Hindley P. Psychiatric aspects of hearing impairments. J Child Psychol Psychiatry. 1997;38(1):101-17.

16. Porto CC, Teixeira CMFS. Linguagem dos órgãos e comunicação médico-paciente. J Bras Med. 2005;89(1):21-3.

17. Jeffrey $D$, Austen $S$. Adapting de-escalation techniques with deaf service users. Nurs Stand. 2005;19(49):41-17.
18. Martin SE, Irwin J. The use of sign language interpreting services by medical staff in Dundee. Health Bull (Edind). 2000;58(3):186-91.

19. Steinberg AG, Wiggins EA, Barmada CH, Sullivan VJ. Deaf women: experiences and perceptions of healthcare system access. J Womens Health (Larchmt). 2002;11 (8):729-41.

20. Tucker BP. Access to health care for individuals with hearing impairments. Houst Law Rev. 2000;37(4):1101-62.

21. Bat-Chava Y, Martin D, Kosciw JG. Barriers to HIV/AIDS knowledge and prevention among deaf and hard of hearing people. AIDS Care. 2005;17(5):623-34.

22. Porter A. Sign-language interpretation in psychotherapy with deaf patients. Am J Psychother. 1999;53(2):163-76.

23. Witte TN, Kuzel AJ. Elderly deaf patient' health care experiences. J Am Board Fam Pract. 2000;13(1):17-22.

24. Barnett S. Cross-cultural communication with patients who use American Sign Language. Fam Med. 2002; 34 (5):376-82

25. Strachan J. The sound barrier. Nurs Stand. 1999;13(25):24

26. McAleer M. Communicating effectively with deaf patients. Nurs Stand. 2006;20(19)51-4.

27. Hines J. Communication problems of hearing-impaired patients. Nurs Stand. 2000;14(19):33-7.

28. Pollard RQ. Professional psychology and deaf people: the emergence of a discipline. Am Psychol. 1996; 51(4):389-96.

29. Stebnicki JA, Coeling HV. The culture of the deaf. J Transcult Nurs. 1999;10(4):350-7.

30. Veet V. Mídia e deficiência: série diversidade. Brasília: Fundação Banco do Brasil; 2003.

31. Raifman LJ, Vernon M. New rights for deaf patients; new responsibilities for mental hospitals. Psychiatr $Q$. 1996;67(3)209-20.

32. Herring R, Hock I. Communicating with patients who have hearing loss. N J Med. 2000;97(2)45-9.

33. Brasil. Decreto n. 5.626, de 22 de dezembro de 2005. Regulamenta a Lei n. 10.436, de 24 de abril de 2002, que dispõe sobre a Língua Brasileira de Sinais - LIBRAS, e o art. 18 da Lei n. 10.098 de 19 de dezembro de 2000 [legislação na Internet]. Brasília; 2005. [citado 2007 jan. 10]. Disponível em: http://200.181.15.9/ ccivil_03/_Ato2004-2006/2005/Decreto/D5626.htm

34. Brasil. Ministério da Saúde. A pessoa com deficiência e o Sistema Único de Saúde. Brasília; 2006.

35. Federação Nacional de Educação e Integração dos Surdos (FENEIS). LIBRAS: Língua Brasileira de Sinais [texto na Internet]. [citado 2007 jan. 10]. Disponível em: http:/ /www.feneis.com.br/page/index.asp

36. Steinberg AG, Barnett $S$, Meador HE, Wiggins EA, Zazove P. Health care system accessibility. Experiences and perceptions of deaf people. J Gen Intern Med. 2006; 21 (3):260-6. 